\title{
Development of the mathematical model of heat and mass transfer for researching the processes of evaporation of inhomogeneous water droplets
}

\author{
Dmitry Antonov ${ }^{1}$, Ivan Voytkov ${ }^{1}$, and Maxim Piskunov ${ }^{1, *}$ \\ ${ }^{1}$ National Research Tomsk Polytechnic University, 634050 Tomsk, Russia
}

\begin{abstract}
On the basis of experimental date the heat and mass transfer models are developed in ANSYS Fluent software package for researching the processes of evaporation of inhomogeneous water droplets. The influence of the temperature of the gases $(550-850 \mathrm{~K})$ on the evaporation of inhomogeneous water droplets is estimated. Times of complete liquid evaporation from free surface of inhomogeneous water droplets and explosive vaporization of water droplets at different gas temperatures are established.
\end{abstract}

\section{Introduction}

In the recent years, in the development of chemical and energy technologies based on inhomogeneous and two-phase gas and vapor-drop streams, much attention is paid to the study of the intensification of heat transfer processes, the establishment of appropriate mechanisms for phase transformations of liquid droplets with solid inclusions, the conditions for realizing different regimes of phase transformations at the "liquid-solid opaque particle" interfaces [1-2]. This approach contributes the development of a large range of practical applications (for example, flame cleaning of liquids [3] and polydisperse firefighting [4]).

The results of investigations $[3,4]$ are shown that the introduction of carbon particles into water droplets intensifies several times the phase transformations of the latter in a hightemperature (more than $700 \mathrm{~K}$ ). It is established [3] that the evaporation of a liquid droplet containing a commensurate opaque inclusion in high-temperature gases is realized according to one of two schemes: 1) evaporation only from the free (external) surface of the drop; 2) evaporation from a free surface and intensive vaporization at internal interfaces. In the experiment, the range of various parameters (temperatures gases, volumes and shapes of droplets) is limited. Therefore, it is expedient to create a mathematical model of heat and mass transfer for studying the evaporation processes of inhomogeneous water droplets.

The aim of this work is development of the mathematical model of heat and mass transfer for researching the processes of evaporation of inhomogeneous water droplets.

\footnotetext{
* Corresponding author: piskunovmv@tpu.ru
} 


\section{Mathematical model}

In the statement of the heat and mass transfer problem we assumed the initial water temperature $T_{\mathrm{w} 0}=300 \mathrm{~K}$, gas temperature $T_{\mathrm{g}}=550-850 \mathrm{~K}$. Inhomogeneous droplets of water are heated due to thermal conductivity when they are flowed by a stream of hightemperature gases.

In the numerical modeling we assumed that the droplets have a time-constant spherical form (Fig. 1), are immobile, and are flown around by a high-temperature gas flow with the known velocity $V_{\mathrm{g}} \approx 0.3 \mathrm{~m} / \mathrm{s}$.

Figure 1 represents schemes of the domains of solution of the heat and mass transfer problems for researching the processes of evaporation of inhomogeneous water droplets.

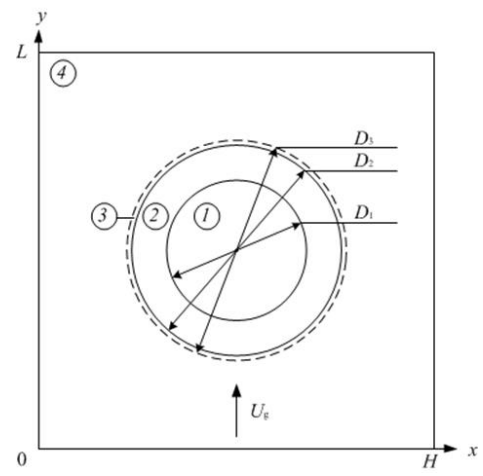

Fig. 1. Scheme of solution area: water + graphite inclusion ( 1 - graphite inclusion; 2 - water liquid; 3 - water vapor; 4 - high-temperature gases).

The system of nonstationary differential equations in partial derivatives for the considered systems within the scope of the assumptions has the following form.

For the vapor-gas mixture $(0<x<H, 0<y<L)$ :

the continuity equation

$$
\frac{\partial u}{\partial x}+\frac{\partial w}{\partial y}=0
$$

the motion equation

$$
\begin{gathered}
\rho_{3}\left(\frac{\partial u}{\partial t}+u \frac{\partial u}{\partial x}+w \frac{\partial u}{\partial y}\right)=-\frac{\partial P_{3}}{\partial x}+v_{3}\left(\frac{\partial^{2} u}{\partial x^{2}}+\frac{\partial^{2} u}{\partial y^{2}}\right) \\
\rho_{3}\left(\frac{\partial w}{\partial t}+u \frac{\partial w}{\partial x}+w \frac{\partial w}{\partial y}\right)=-\frac{\partial P_{3}}{\partial y}+v_{3}\left(\frac{\partial^{2} w}{\partial x^{2}}+\frac{\partial^{2} w}{\partial y^{2}}\right)+\rho_{3} \beta g\left(T_{3}-T_{0}\right) .
\end{gathered}
$$

the energy equation

$$
\rho_{3} C_{3}\left(\frac{\partial T_{3}}{\partial t}+u \frac{\partial T_{3}}{\partial x}+w \frac{\partial T_{3}}{\partial y}\right)=\lambda_{3}\left(\frac{\partial^{2} T_{3}}{\partial x^{2}}+\frac{\partial^{2} T_{3}}{\partial y^{2}}\right) .
$$

the water vapor diffusion equation 


$$
\rho_{2}\left(\frac{\partial C_{\mathrm{w}}}{\partial t}+u \frac{\partial C_{\mathrm{w}}}{\partial x}+w \frac{\partial C_{\mathrm{w}}}{\partial y}\right)=\rho_{2} D_{2}\left(\frac{\partial^{2} C_{\mathrm{w}}}{\partial x^{2}}+\frac{\partial^{2} C_{\mathrm{w}}}{\partial y^{2}}\right) .
$$

the balance equation

$$
C_{\mathrm{g}}+C_{\mathrm{w}}=1
$$

For water droplets $\left(0<r<R_{\mathrm{d}}, 0<\varphi<2 \pi\right)$ :

the equation of thermal conductivity in the spherical system of coordinates

$$
\frac{\partial T_{1}}{\partial t}=a_{2}\left[\frac{1}{r^{2}} \frac{\partial}{\partial r}\left(r^{2} \frac{\partial T_{1}}{\partial r}\right)+\frac{1}{r^{2} \sin (\varphi)} \frac{\partial}{\partial \varphi}\left(\sin (\varphi) \frac{\partial T_{1}}{\partial \varphi}\right)\right] .
$$

Initial conditions $(t=0): T=T_{0}, C_{\mathrm{w}}=0, C_{\mathrm{g}}=1, u=w=0, P=P_{0}$ при $0<x<H, 0<y<L ; T=T_{0}$ at $0<r<R_{\mathrm{d}}, 0<\varphi<2 \pi$.

Boundary conditions at $t>0$ :

$$
\begin{aligned}
& T=T_{\mathrm{g}}, C_{\mathrm{w}}=0, C_{\mathrm{g}}=1, V=V_{\mathrm{g}} \text { at } y=0,0<x<H ; \\
& \frac{\partial^{2} T}{\partial y^{2}}=0, \frac{\partial^{2} C_{\mathrm{w}}}{\partial y^{2}}=0, \frac{\partial^{2} C_{\mathrm{g}}}{\partial y^{2}}=0 \text { at } y=L, 0<x<H ; \\
& \frac{\partial T}{\partial t}=0, C_{\mathrm{w}}=0, C_{\mathrm{g}}=0 \text { at } x=0,0<y<L ; \\
& \frac{\partial T}{\partial t}=0, C_{\mathrm{w}}=0, C_{\mathrm{g}}=0 \text { at } x=H, 0<y<L .
\end{aligned}
$$

Boundary conditions for "liquid - gas" were set taking into account vaporization:

$$
R=R_{1}, 0<\varphi<2 \pi, \lambda_{1} \frac{\partial T_{1}}{\partial R}=\lambda_{2} \frac{\partial T_{2}}{\partial R}+W_{e 2} Q_{\mathrm{e} 2}, \rho_{2} D_{2} \frac{\partial C_{w}}{\partial r}=W_{e 2} .
$$

The components of the velocity vector $(u$ and $w)$ are determined using the following equations:

$$
u=\left(\frac{2}{\rho_{3}} \frac{\partial P}{\partial x}\right)^{1 / 2}, w=\left(\frac{2}{\rho_{3}} \frac{\partial P}{\partial y}\right)^{1 / 2}
$$

We used steps in time $\Delta t=0.1 \mathrm{~s}$ and in coordinate grid $\Delta x=\Delta y=0.05 \mathrm{~mm}$. Near the phase transition boundaries the coordinate grid became thicker (up to $\Delta x=\Delta y=\Delta r=0.001 \mathrm{~mm}$ ).

\subsection{Nomenclature}

$a$ - temperature conductivity, $\mathrm{m}^{2} / \mathrm{s} ; C$ - specific heat capacity, $\mathrm{J} /(\mathrm{kg} \cdot \mathrm{K}) ; D$ - diffusion coefficient, $\mathrm{m}^{2} / \mathrm{s} ; C_{\mathrm{g}}-$ concentration of combustion products $\left(0<C_{\mathrm{g}}<1\right) ; C_{\mathrm{w}}-$ concentration of water vapor; $g-\quad$ acceleration of gravity, $\mathrm{m} / \mathrm{s}^{2} ; H, L-$ dimensions of solution area, $\mathrm{mm} ; P$ - pressure, $\mathrm{N} / \mathrm{m}^{2} ; R_{\mathrm{d}}$ - initial droplet radius, $\mathrm{mm} ; t$ - time, $\mathrm{s} ; T$ - temperature, $\mathrm{K}$; $T_{0}$ - initial temperature, $\mathrm{K} ; T_{\mathrm{g}}$ - temperature of gases, $\mathrm{K} ; Q_{\mathrm{e}}$ - water vaporization heat, $\mathrm{J} / \mathrm{kg}$; 
$W_{\mathrm{e}}$ - evaporation rate, $\mathrm{kg} /\left(\mathrm{m}^{2} \cdot \mathrm{s}\right) ; V_{\mathrm{g}}$ - velocity of gases (combustion products), $\mathrm{m} / \mathrm{s}$; $x, y$ - coordinates of the Cartesian coordinate system, $\mathrm{m} ; u, w-x$ and $y$ components of convection velocity of the gas-vapor mixture, $\mathrm{m} / \mathrm{s} ; \Delta t$ - time step size, $\mathrm{s} ; \Delta x, \Delta y$, $\Delta r$ - coordinate steps, $\mathrm{mm} ; \rho-$ density, $\mathrm{kg} / \mathrm{m}^{3} ; \lambda-$ thermal conductivity, $\mathrm{W} /(\mathrm{m} \cdot \mathrm{K})$; $v$ - kinematic viscosity, $\mathrm{m}^{2} / \mathrm{s} ; \beta$ - thermal expansion coefficient, $\mathrm{K}^{-1} ; r, \varphi$ - coordinates of the Spherical coordinate system, m; indices: 1 - water droplets, 2 - water vapor, 3 - gasvapor mixture.

\section{Results}

Figures 2 represents typical temperature fields of the vapor-gas mixture for the above statements. Simulations are estimated for droplets with volume 5, 10 and $15 \mathrm{mkl}$.
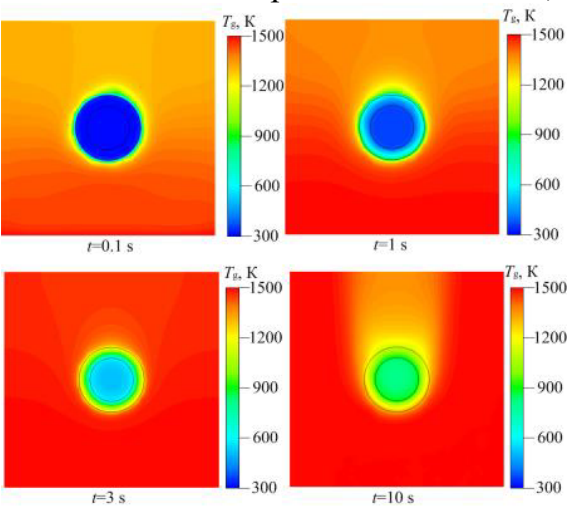

Fig. 2. Temperature fields for inhomogeneous water droplet (water + graphite inclusion) for different times at $T_{\mathrm{g}}=1500 \mathrm{~K}$ (volume of droplet $V_{\mathrm{d}}=10 \mathrm{mkl}$ ).

The results of numerical studies show the dependence of the times of complete evaporation (Fig.3) and the explosive vaporization (Fig.4) of inhomogeneous water droplets at different gas temperatures $\left(T_{\mathrm{g}}=550-850 \mathrm{~K}\right)$. A satisfactory correlation of the results of numerical studies with experiment is shown.

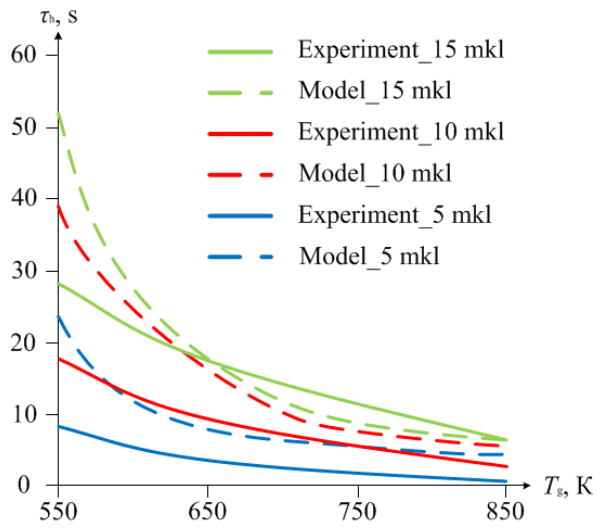

Fig. 3. Times of complete liquid evaporation $\tau_{\mathrm{h}}$ from free surface of inhomogeneous water droplets at different gas temperatures. 


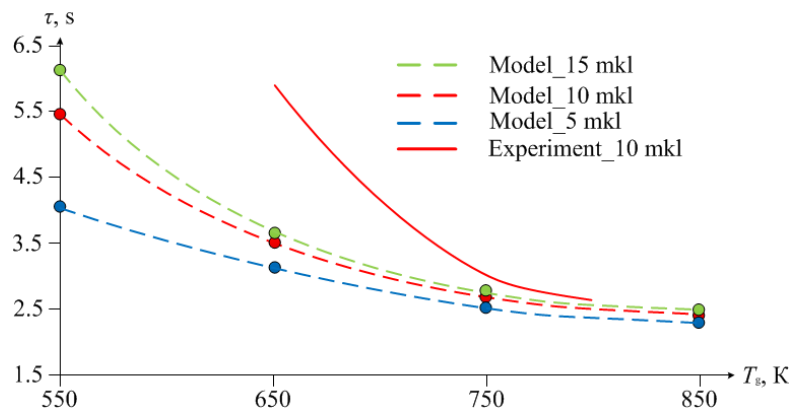

Fig. 4. Times of explosive vaporization of water droplets $\tau$ at different gas temperatures.

The obtained dependences (Fig.3) illustrate, as it was possible to assume, with the increasing gas temperature the evaporation of the liquid is more intensively. It should be noted that when the inhomogeneous droplets of water are heated in a gas with temperature above $650-850 \mathrm{~K}$, the effect of their "explosive" destruction with "boiling" of the liquid and the decay of the film into smaller droplets is recorded. Such an effect arises from the considerable warming up of the solid particle on the side of contact with the gases, as well as due to the transmission of the energy radiation from the high-temperature gases to the internal interface of the "inclusion-liquid". The destruction of an inhomogeneous droplet was preceded by evaporation from the free surface of the liquid film, formation, a significant increase in volume, and the fusion of vapor bubbles at the internal interface. The presented times of explosive vaporization of inhomogeneous water droplets at the temperature gases (Fig. 4) illustrate that the times of explosive vaporization decrease with increasing temperature.

\section{Conclusion}

Based on the results of the experiments, a mathematical model of heat and mass transfer in the Ansys Fluent package has been developed, which can be used to predict the times of complete evaporation and the times of explosive vaporization of inhomogeneous water droplets. A satisfactory correlation between simulation results and experimental results is shown.

Research was supported by Foundation of President of Russian Federation (MD-1221.2017.8).

\section{References}

1. D.H. Doh, J.H. Yum, G.R. Cho, M.H. Kim, G.W. Ryu, M. Takei, J. Therm. Sci. 22, 5 (2013)

2. I. Gherasim, G. Roy, C.T. Nguyen, D. Vo-Ngoc, Int. J. Therm. Sci. 50, 3 (2011)

3. D.V. Antonov, R.S. Volkov, M.V. Piskunov, P.A. Strizhak, Technical Physics Letters, 42, 3 (2016)

4. R.S. Volkov, G.V. Kuznetsov, P.A. Strizhak, Int. J. Therm. Sci. 88, 1 (2015) 